\title{
Spatio-temporal spillover risk of yellow fever in Brazil
}

\author{
RajReni B. Kaul ${ }^{1,2^{*}+} \mathbb{D}$, Michelle V. Evans ${ }^{1,2+}$, Courtney C. Murdock ${ }^{1,2,3,4,5,6}$ and John M. Drake ${ }^{1,2}$
}

\begin{abstract}
Background: Yellow fever virus is a mosquito-borne flavivirus that persists in an enzoonotic cycle in non-human primates (NHPs) in Brazil, causing disease in humans through spillover events. Yellow fever (YF) re-emerged in the early 2000s, spreading from the Amazon River basin towards the previously considered low-risk, southeastern region of the country. Previous methods mapping YF spillover risk do not incorporate the temporal dynamics and ecological context of the disease, and are therefore unable to predict seasonality in spatial risk across Brazil. We present the results of a bagged logistic regression predicting the propensity for YF spillover per municipality (administrative sub-district) in Brazil from environmental and demographic covariates aggregated by month. Ecological context was incorporated by creating National and Regional models of spillover dynamics, where the Regional model consisted of two separate models determined by the regions' NHP reservoir species richness (high vs low).

Results: Of the 5560 municipalities, 82 reported YF cases from 2001 to 2013. Model accuracy was high for the National and low reservoir richness (LRR) models (AUC $=0.80$ ), while the high reservoir richness (HRR) model accuracy was lower (AUC $=0.63$ ). The National model predicted consistently high spillover risk in the Amazon, while the Regional model predicted strong seasonality in spillover risk. Within the Regional model, seasonality of spillover risk in the HRR region was asynchronous to the LRR region. However, the observed seasonality of spillover risk in the LRR Regional model mirrored the national model predictions.

Conclusions: The predicted risk of YF spillover varies with space and time. Seasonal trends differ between regions indicating, at times, spillover risk can be higher in the urban coastal regions than the Amazon River basin which is counterintuitive based on current YF risk maps. Understanding the spatio-temporal patterns of YF spillover risk could better inform allocation of public health services.
\end{abstract}

Keywords: Yellow fever, Risk mapping, Vectors, Brazil, Spatial epidemiology, Arboviruses

\section{Background}

Yellow fever virus (YFV), a mosquito-borne flavivirus, began re-emerging in Brazil in the early 2000s, gradually spreading from the Amazon River basin towards the southeastern region of the country [1,2]. Unlike other mosquito-borne diseases in Brazil, such as malaria and dengue, YFV circulates in a sylvatic transmission cycle among non-human primate (NHP) reservoirs maintained by Haemagogus and Sabethes mosquitoes, with spillover occurring when mosquitoes transmit outside

\footnotetext{
* Correspondence: reni@uga.edu

${ }^{\dagger}$ RajReni B. Kaul and Michelle V. Evans contributed equally to this work.

${ }^{1}$ Center for the Ecology of Infectious Diseases, University of Georgia, Athens, GA, USA

${ }^{2}$ Odum School of Ecology, University of Georgia, Athens, GA, USA

Full list of author information is available at the end of the article
}

this cycle to human hosts [3, 4]. Thus, YFV spillover events leading to human yellow fever (YF) disease are closely tied to population dynamics and community composition of both mosquitoes and NHP. Beginning in late 2016, Brazil experienced a YF outbreak of over 450 cases, more cases than had been reported in the previous fifteen years combined [5]. The majority of these cases were outside the previously defined range of YF and near densely populated, urban areas. To date, the Brazilian Ministry of Health classifies these cases as "sylvatic", or the direct result of a spillover event due to transmission of YFV from local NHP reservoirs to humans via Haemagogus and Sabethes mosquitoes. The unexpected emergence of YF in these regions previously considered low-risk highlights the need for a better

(C) The Author(s). 2018 Open Access This article is distributed under the terms of the Creative Commons Attribution 4.0 International License (http://creativecommons.org/licenses/by/4.0/), which permits unrestricted use, distribution, and 
understanding of the spatial and temporal patterns of YF spillover risk.

Infectious disease mapping is one such method to predict spatial patterns in disease transmission risk [6]. Predictive maps of disease risk use statistical methods to account for disease transmission dynamics via their relationship with environmental and socio-demographic covariates. This approach has been applied to a range of infectious diseases, including Ebola [7, 8], malaria [9, 10] and dengue $[11,12]$. Past efforts at mapping YF have focused primarily on environmental variables as drivers of YF spillover dynamics [13-15]. However, these studies have relied on static environmental variables, ignoring multi-year and annual cycles in environmental conditions and spillover events which occur on seven and fourteen year cycles $[16,17]$. Multi-year cycles are due to the El Niño-Southern Oscillation [18] and the accumulation of susceptible hosts in NHP populations [16]. Spillover events are also seasonal, with the majority of cases occurring during the rainy season (approximately December-April), when mosquito vector abundances are highest [19]. Predictive models that focus on a snapshot in time, or a normalized average of climate data, are therefore unable to capture temporal changes in spillover risk.

Because the compositions of mosquito and NHP communities vary over space, it might be that the processes and corresponding environmental drivers of YF spillover are dependent on the underlying ecological context. For example, in the West Nile virus (WNV) system in the USA, WNV is positively associated with urban land cover in the northeast and with agricultural land cover in the west, due to different habitat preferences of vectors of the disease, which differ across regions [20]. Like the USA, Brazil contains multiple biomes, with Amazon rainforest in the northwestern region, cerrado (savanna) in the central region, and Atlantic rainforest in coastal areas in the southeast. We reasoned that the processes and corresponding environmental drivers of YF spillover are dependent on the underlying ecological context or region. Multiple models, unlike a single model, can incorporate different directions and magnitudes of relationships between environmental variables and YF spillover. By assuming a single homogeneous process of spillover across the country, current models of spatial risk of YF spillover do not allow for the existence of a variety of spillover processes, and therefore cannot identify their corresponding environmental drivers.

Past spatial mapping of YF risk in Brazil has assumed a temporally constant risk or similar environmental drivers of YF spillover across all regions of Brazil, regardless of their NHP species richness [14, 15, 21, 22]. Here, we first present a statistical model (National model) that incorporates monthly variation in covariates to predict the propensity of YF spillover across municipalities (sub-state administrative units) of Brazil. Secondly, we better contextualize the spillover process by fitting models to two contiguous regions determined by a natural break in NHP reservoir species richness. The composite of these models are presented as the Regional model. Finally, we present the models' predictions of the spatio-temporal risk of YF transmission by month across Brazil from 2001 to 2013 for the National and Regional models.

\section{Methods}

YF spillover propensity was estimated for each month between January 2001 and December 2013 using 12 environmental and socio-demographic covariates for each municipality by a bagged logistic regression model (Fig. 1, Table 1). The spatial and temporal unit of the model, municipality and month (hereby referred to as municipality-month) was based on the finest resolution of epidemiological data reported by the Brazilian Ministry of Health. Municipality-months with at least a single reported YFV case were given a positive label for the binary response variable, spillover event. Data collection methods are briefly reported here, with additional details reported in Additional file 1.

\section{Data collection}

We chose environmental and socio-demographic covariates that are suspected drivers of YF disease transmission by either modifying the host population, mosquito population or host proximity to competent non-human primate (NHP) reservoirs (Table 1). Variables were downloaded at the monthly, yearly, or static temporal resolution, as determined by the availability of data for the whole country of Brazil. In the case where annual values were the smallest temporal resolution, values were held constant for the year. Variables were spatially averaged by area to the resolution of the municipality to provide a mean value for each municipality-month. Aggregating spatial variables can lead to bias (e.g. the modifiable areal unit problem) by analyzing the relationship between variables at a coarser spatial scale than the process of spillover occurs [23, 24]. However, we limited bias introduced by aggregation by conducting our analysis at the finest grain possible given the availability of epidemiological data. Further, we believe conducting an analysis at the scale of the municipality rather than pixel-level is more relevant to public health decision-making as this corresponds to the smallest administrative level. All variables were visually inspected for normality and cube root transformed if needed, except for population density, which was $\log _{10}$-transformed (Table 1). 


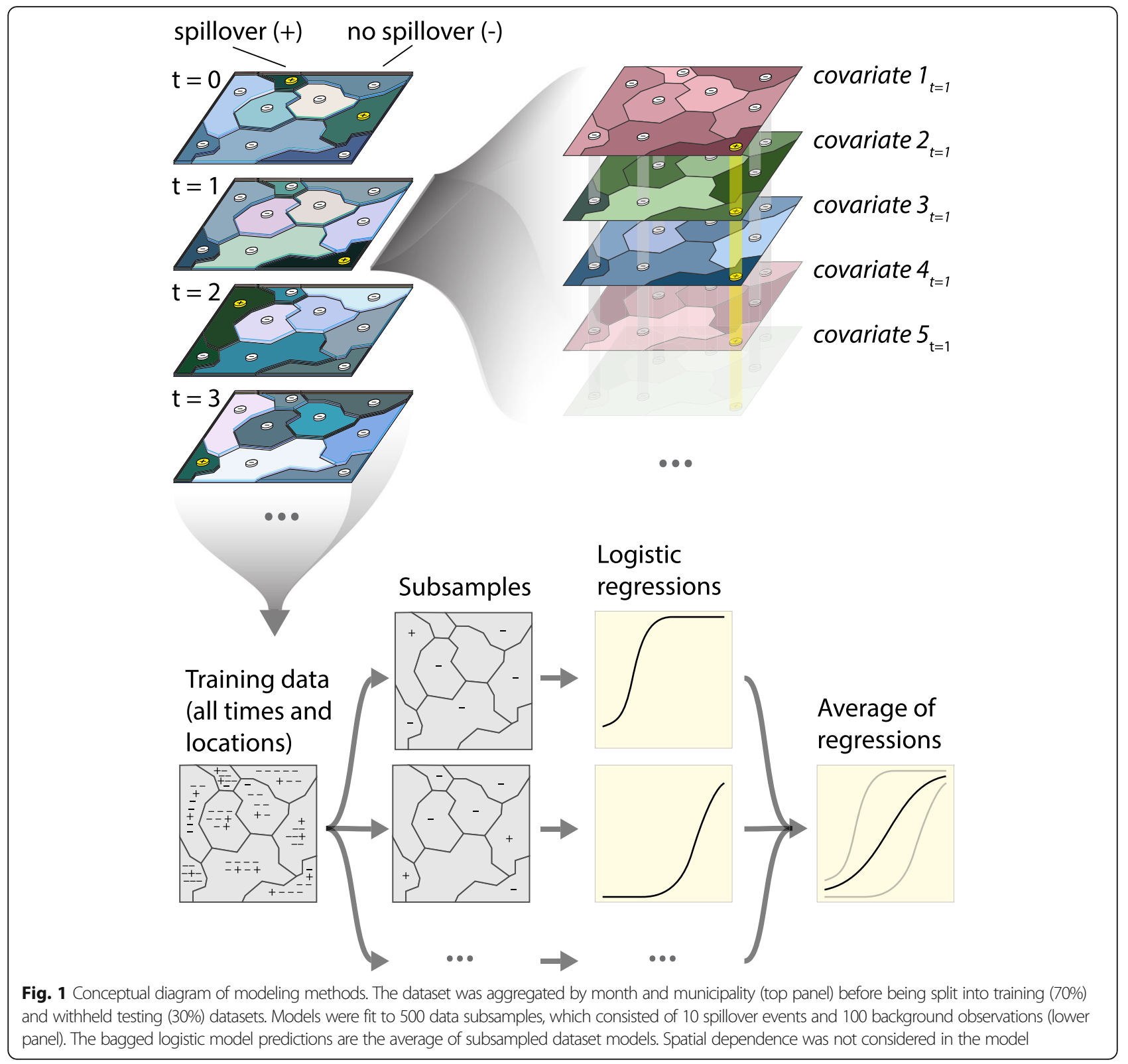

Mosquitoes are extremely sensitive to changes in temperature and relative humidity, and their abundance is often correlated with rainfall [12, 25, 26]. We therefore chose mean monthly temperature (hereby referred to as temperature), the Normalized Difference Vegetation Index (NDVI), and mean hourly rainfall averaged over a month (hereby referred to as rainfall) as variables relevant to mosquito population dynamics. While daily temperature variability and extremes are important for mosquito dynamics $[27,28]$, environmental variables are not available at this fine of a temporal-scale for the entire country of Brazil. We therefore used monthly averages to represent the climatic profile of a municipality corresponding to the temporal resolution of the epidemiological reports. Preliminary data analysis revealed spatial minima, maxima and mean variables to be highly correlated $(\rho>0.90$, Additional file 1 : Figure S1). Univariate analyses on the training data found no difference in explanatory power amongst variables (Additional file 1: Table S1). Therefore, we chose the spatial mean to represent each environmental variable to reduce bias due to collinearity [29]. In addition to environmental variables that can influence mosquito abundances, we also included the species ranges of mosquito vectors of YF in our model. We estimated the occurrence of mosquito vectors from published MaxEnt models of distributions of three YF vector species (Hg. leucocelaenus, Hg. janthinomys and $\mathrm{Sa}$. chloropterus). The maximum probability amongst the three species was chosen for each municipality, 
Table 1 Summary of data sources used in the model (see Additional file 1 for additional information on variable collection)

\begin{tabular}{|c|c|c|c|c|}
\hline Data type & Temporal resolution & Raw spatial resolution & Source & Extreme variable? \\
\hline Yellow fever incidence & Monthly & Municipality & MS & - \\
\hline Population density & Yearly & Municipality & MS & - \\
\hline Land surface temperature & Monthly & $0.05^{\circ}$ & LPDAAC & Yes \\
\hline Normalized difference vegetation index & Monthly & $1 \mathrm{~km}$ & LPDAAC & Yes \\
\hline Average hourly rainfall ${ }^{a}$ & Monthly & $0.25^{\circ}$ & TRMM & Yes \\
\hline Fire density ${ }^{a}$ & Monthly & $1 \mathrm{~km}$ & FIRMS & Yes \\
\hline Non-human primate species richness ${ }^{a}$ & Static & Municipality & IUCN & - \\
\hline Agricultural and non-human-primate overlap ${ }^{a}$ & Yearly & $1 \mathrm{~km}$ & IUCN/ LPDAAC & - \\
\hline Maximum probability of mosquito vector occurrence & Static & $0.04167^{\circ}$ & VectorMap & - \\
\hline
\end{tabular}

Abbreviations: MS Brazilian Ministry of Health, LPDAAC NASA Land Processes Distributed Active Archive Center, TRMM Tropical Rainfall Monitoring Mission, FIRMS Fire Information for Resource Management System, IUCN International Union for Conservation of Nature

a Variable was cube root transformed prior to model construction

resulting in the maximum probability of a mosquito vector occurring within that municipality.

Transmission from the sylvatic cycle to human hosts occurs when human hosts are in proximity to NHP reservoirs of the pathogen. Human proximity to NHPs was captured in three variables. First, we created a variable of fire density, as a representation of anthropogenic land conversion [30]. Secondly, we calculated the species richness of NHPs susceptible to YF per municipality to represent reservoir abundance. In general, host species richness positively correlates with spillover events of diseases of zoonotic origin [31]. Other factors, such as species' competences, relative abundances, and overall community structure, can further influence spillover risk $[32,33]$. However, the role of individual species in YF transmission and the population abundances of NHPs across all of Brazil is not known. Finally, as a proxy for longer-term NHP-human contact, we calculated the proportion of a municipality's land area that is both agricultural land use and within a primate species' range. To calculate this proportion of agricultural land overlapping NHP species' ranges, we overlaid species range maps with land cover maps of agricultural land use. We then calculated the proportion of total municipality area that was both in agricultural use and within a genus range and summed across all nine genera, resulting in a value of $0-9$ per municipality per year.

Additional variables were constructed to capture extreme events, which are considered to be triggers for socio-ecological events like YF spillovers. The three environmental covariates intended to reflect mosquito dynamics (temperature, NDVI and rainfall) along with fire density were scaled to the maximum value for that specific calendar month and municipality. The inclusion of anomalies allowed us to distinguish between seasonal trends and particularly extreme events acting as triggers [7].

Human host population dynamics were captured by annual estimates of human population by municipality, obtained from the Brazilian Institute of Geography and Statistics. This was converted to population density based on municipality area, and then $\log _{10}$-transformed. Higher frequency population density estimates or proportion of the population vaccinated were not available by municipality.

Finally, a spillover occurred in a municipality-month that had one or more confirmed YF cases. We used the monthly confirmed cases of YF reported by the Brazilian Ministry of Health Notification of Injury Information System (Sianan Net) to determine whether a spillover event occurred for each municipality in each of the 156 months between January 2001 and December 2013. Exploratory analysis indicated the majority of YF cases were single cases per municipality-month (mean $1.83 \pm$ 2.46 YF cases per municipality-month, Additional file 1: Figure S2). Given the consistent reporting of single cases per municipality-month, we collapsed the continuous case counts data into the binary spillover variable. In municipality-months with multiple cases reported, the binary classification assumes that at least one case is the result of sylvatic transmission. Municipality-months with a single case and those with multiple cases are equally weighted as a single spillover event.

The compiled dataset had 867,360 observations (5560 municipalities times 156 months), 106 of which were positive for a spillover event (Table 2). No additional variable selection was conducted before model training and testing.

\section{Predictive model}

We used a bagged logistic regression to predict the monthly propensity of YF spillover per municipality across Brazil, which we refer to as the "National model" (Fig. 1). Bagging (bootstrap aggregating) is an ensemble machine learning approach that combines the predictive power of many less informative models to provide an overall more accurate prediction [34, 35]. The less 
Table 2 Dataset summary. Training and testing dataset used to build the National model, which was then subset into the low reservoir richness (LRR), and high reservoir richness (HRR) Regional models

\begin{tabular}{|c|c|c|c|c|c|c|}
\hline \multirow[t]{2}{*}{ Model } & \multicolumn{2}{|c|}{ Training dataset } & \multicolumn{2}{|c|}{ Testing dataset } & \multicolumn{2}{|c|}{ Whole dataset } \\
\hline & Positive & Background & Positive & Background & Positive & Background \\
\hline National & 74 & 607,077 & 32 & 260,177 & 106 & 867,254 \\
\hline LRR & 59 & 584,263 & 27 & 250,251 & 86 & 834,514 \\
\hline HRR & 15 & 22,814 & 5 & 9926 & 20 & 32,740 \\
\hline
\end{tabular}

informative models are constructed from random small subsets of the full dataset. The final model consists of an average of the less informative models. Bagging is also particularly robust with noisy or sparse data, ensuring models are not over fit to the relatively few positive observations [34].

We used a balanced spatial and temporal sampling design via the BalancedSampling package in $\mathrm{R}$ to split the data into training $(70 \%)$ and testing $(30 \%)$ sets, preserving the proportion of municipality-months with a spillover event (positive observations) in each [36]. This sampling design ensured there was no spatial or temporal trend in our training and testing data, which is particularly important given the severe imbalance of positive to negative or background observations (positive observations are $\approx 0.01 \%$ of the dataset) [37]. The training data were used to fit 500 logistic regression models with main effects of the 12 variables. Each logistic regression model was fit to a randomly sampled without replacement subsample of data consisting of 10 and 100 positive and background points in the training dataset, respectively. The final bagged logistic regression model is the average of the logistic regressions.

The data used for the national model above were further divided into two regional datasets, based on species richness of non-human primate reservoirs. These datasets were used to build separate high reservoir richness (HRR, > 5 NHP reservoir species) or low reservoir richness (LRR, $\leq 5$ NHP reservoir species) models, both of which still included NHP richness as a covariate. We refer to the combination of these models as the "Regional model". This division was determined by a natural break in the distribution of the species richness data, which generally corresponded with a geographic break (Fig. 2). In a few instances, municipalities with a low reservoir richness ( $\leq 5 \mathrm{NHP}$ reservoir species) were included in the HRR region, and vice versa to create two contiguous regions. This data driven delineation of regions within the Regional model allowed for potentially different relationships between environmental covariates and spillover risk in the HRR and LRR regions while preserving the maximum number of positive observations within the datasets.

We calculated the performance of each model via the area under the receiver-operator curve (AUC) of the withheld testing data. The AUC represents the model's classification performance and can range from 0.0 to 1.0, where a value of 0.5 is the equivalent of random guessing while an AUC of 1.0 indicates a perfect prediction [38]. Relative variable importance was assessed by the difference in median AUC of 100 permutations to the AUC of the original model, scaled to the largest decrease in AUC due to permutation of a single variable.

\section{Results}

Eighty-two of 5560 municipalities reported YF cases from 2001 to 2013. The number of repeated spillover events in a single municipality was low. Roughly $77 \%$ (63) of municipalities reported cases in only a single month, $18 \%$ (15) reported cases in two months during the 13 year period. Only 3 municipalities reported cases in 3 months, and a single municipality reported cases in four months. Municipalities with more than 2 spillover events were all located Minas Gerais, a southeastern

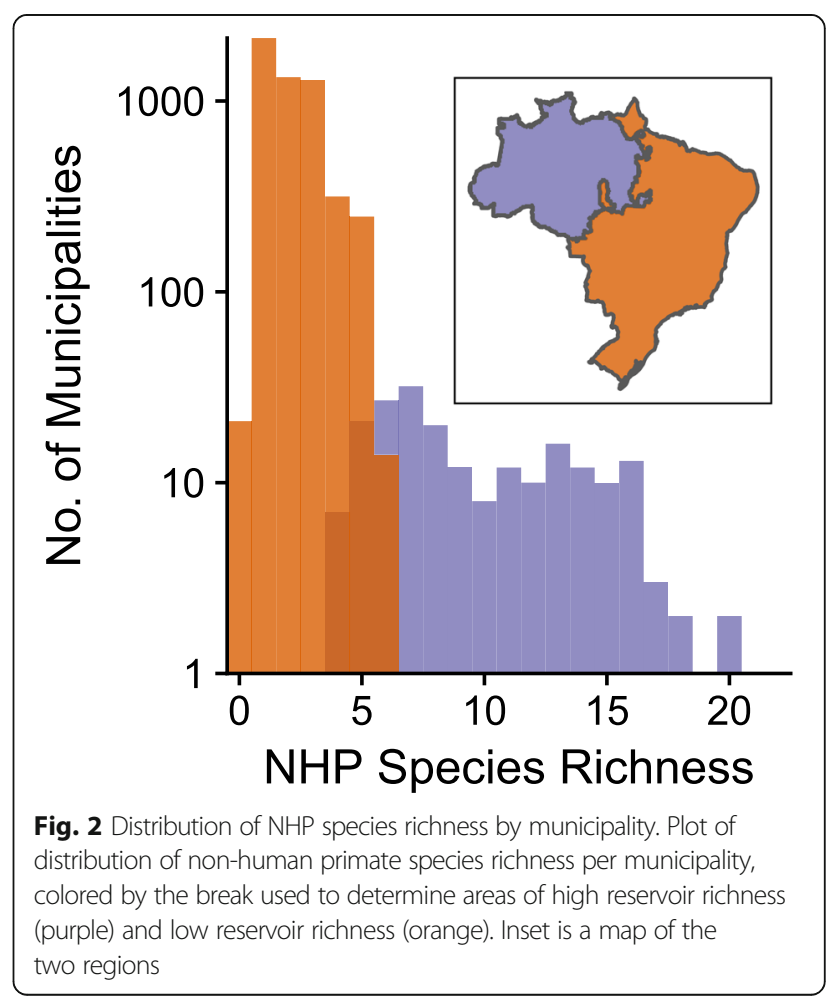


state with locally-acquired cases in the 2017 and 2018 outbreaks.

Model predictive accuracy was high for all three datasets. The AUC values for models evaluated on the training dataset were $0.81,0.79$ and 0.88 for the national dataset, LRR and HRR regions, respectively. When evaluated on the testing dataset, the AUC was 0.80 for both the National and LRR Regional models, while the predictions on the HRR testing dataset had an AUC of 0.63.

The predicted risk of YF spillover varied by season, generally peaking in January for the National and LRR Regional models (Fig. 3). The temporal risk in the HRR model was asynchronous to the other two models, and peaked in May (Fig. 4f). A supplemental movie shows the predicted risk of YF spillover for the entire time series (Additional file 2). The municipalities along the Amazon River and surrounding São Luís, Rio de Janeiro and São Paulo had the greatest change in spillover risk throughout the year (Fig. $4 \mathrm{a}-\mathrm{c}$ ). This annual variation was more pronounced in the Regional models.

We conducted a slope test for each municipality from 2001 to 2013 to explore long-term trends in the predicted intensity of yellow fever spillover. The majority of municipalities saw no long-term trend in predicted intensity over the 156 months included in the dataset (National model:
5028 of 5560 municipalities; Regional model: 5045 of 5560 municipalities). More municipalities experienced a decrease in predicted spillover intensity (National: 489; Regional: 482) than experienced an increase in intensity (National: 43; Regional: 33). Intensity tended to increase over the study period in municipalities along the Amazon River and in the southeastern region of Brazil and decrease along the coast (Additional file 1: Figure S4). When considering calendar months individually for the Regional model, predicted spillover intensity increased the most in July and August between 2000 and 2013, particularly in the southeastern region of the country (Additional file 1: Figure S5), however these months still had the lowest spillover intensity over all. When averaging across municipalities, the highest predicted annual risk was in 2008, followed by 2001.

The area of highest risk differed by model. In the National model, the HRR region had high predicted values of YF spillover risk across all 156 months and the LRR region experienced seasonal shifts in spillover intensity. In contrast, the Regional model predicted high risk of spillover in municipalities near the Amazon River, with lower risk in the higher elevation areas near Brazil's northern border. As in the National model, the LRR region in the Regional model had seasonal variability in

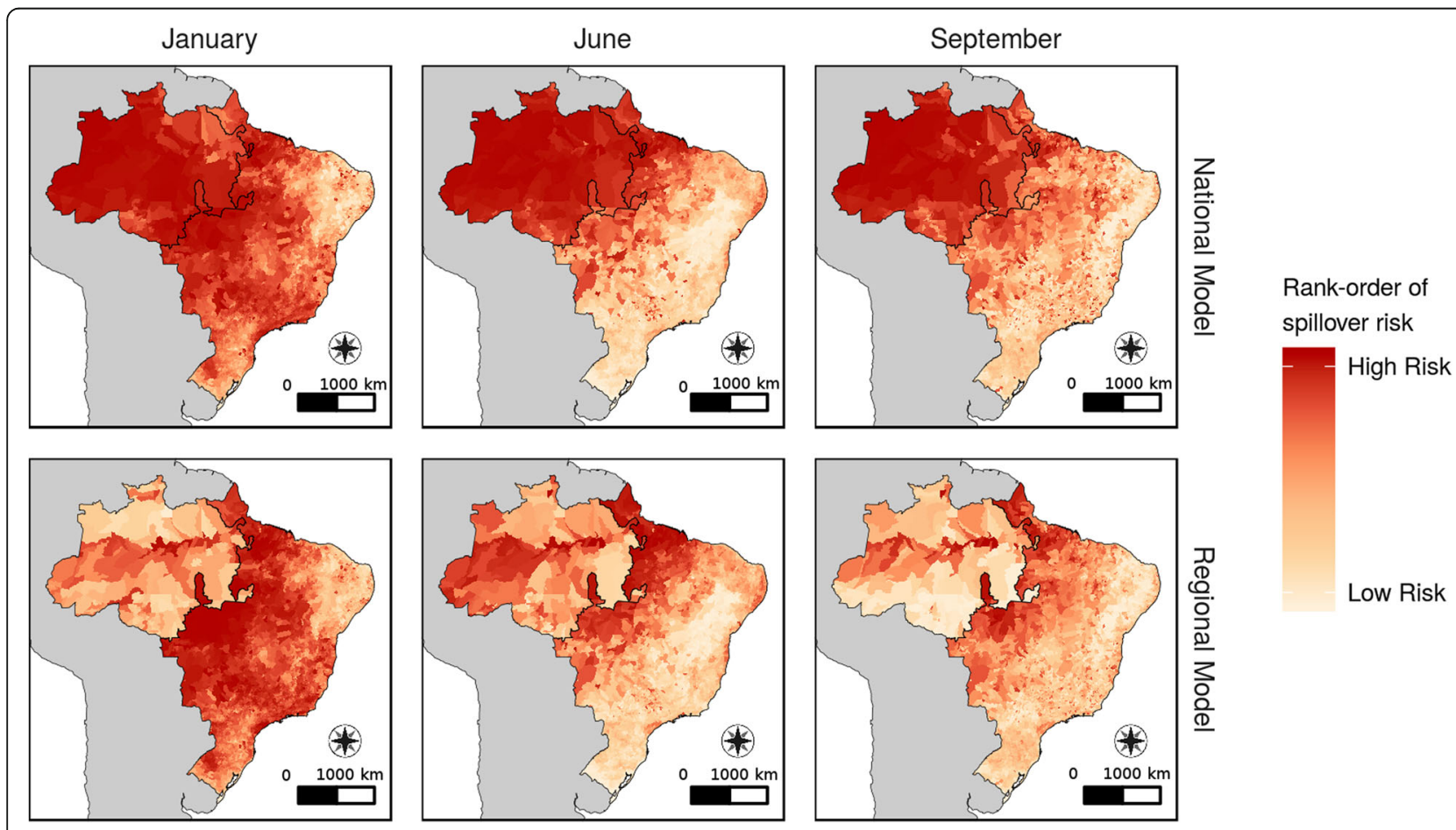

Fig. 3 Predicted spatial risk of yellow fever spillover. Propensity of yellow fever spillover in January, June, and September of 2008. Raw outputs of the model for each municipality-month are rank-ordered to allow for comparison across models. Results from the National model are on the top row and the Regional model are on the bottom row. Black outline represents the split between HRR (northwest) and LRR (southeast) regions. The outline in the national model is for reference only. See supplemental video for entire time series. Map projection: SAD69 Brazil Polyconic. Data source: 2001 municipality boundaries, Brazilian Institute of Geography and Statistics 


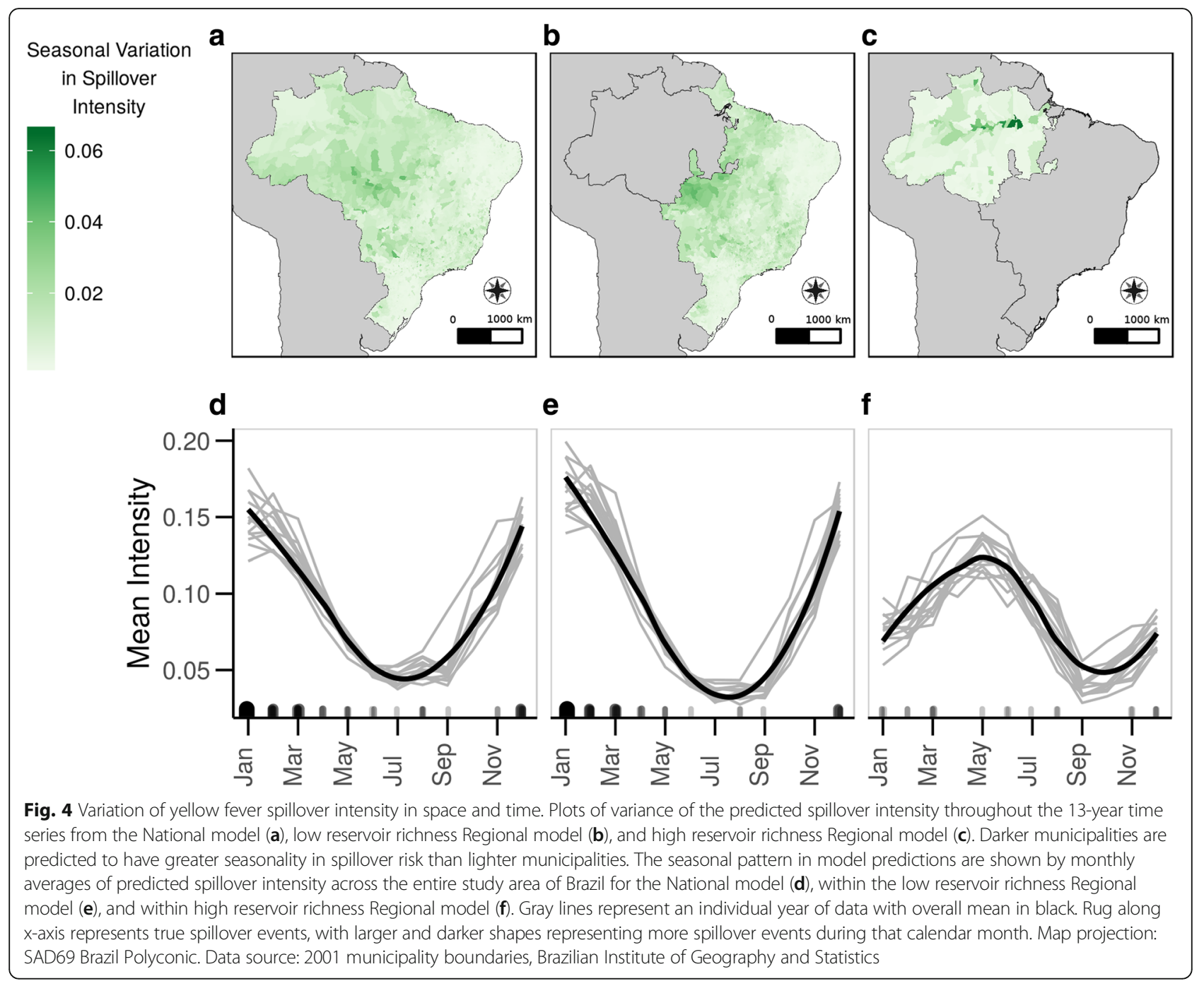

the risk of YF spillover, with a higher risk of spillover from November to March.

The ranked order of variable importance differed between the models (Fig. 5). However, NHP richness was consistently highly ranked. The LRR Regional model and the National model had a similar rank order of variable importance, sharing the top three most important variables (rainfall, temperature, and NHP richness). This is perhaps expected given that the LRR dataset is $96 \%$ of the national dataset. Anomalous conditions as captured in the scaled covariates are of greater importance in the HRR Regional model than the other two models. However, the type of anomalous condition influences the covariates' importance. Scaled rainfall is ranked in the top half of the ranked variables for the National and LRR Regional models, but is less important in the HRR Regional model. The scaled covariates of temperature and fire density follow the opposite pattern, increasing in importance for the LRR Regional model. To a lesser extent, the scaled NDVI follows the same pattern as the anomalous temperature and fire density covariates. The largest change in the rank order between the three models was rainfall (difference of 8 ), followed by scaled rainfall and scaled fire density (tied with a difference of $7)$, then NDVI and vector occurrence (tied with a difference of 6).

The central tendencies of the relative variable importance, as measured by $\triangle \mathrm{AUC}$, for the three models included a natural break in the variables (Fig. 6). The top three variables in the National model (rainfall, NHP richness and temperature) were similar, while the first and second ranked variables for the LRR Regional model (temperature and rainfall) were very similar. In the HRR Regional model, the highest ranked variable (NHP richness) was substantially more important than the second through fourth variables (NDVI, fire density and scaled fire density), which were clustered. However, in general the $\triangle \mathrm{AUC}$ for any variable permutation was small (less than 0.05 decrease from model AUC) indicating that model performance is not driven by a single covariate. 


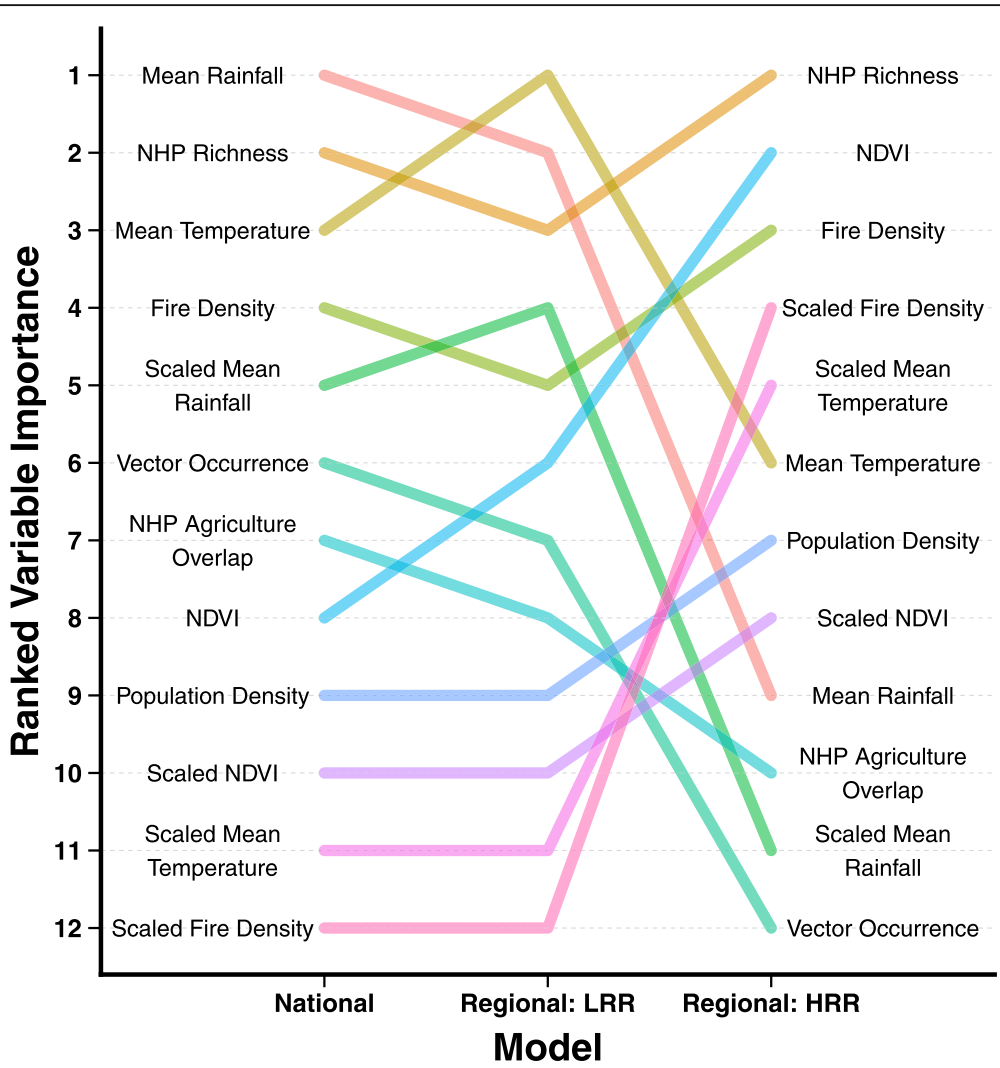

Fig. 5 Rank order of median variable importance. The median variable importance was calculated for the National, low reservoir richness (LRR), and high reservoir richness (HRR) Regional models based on 100 permutations per variable within a model. The variables were ranked from most important (1) to least important (12) for model accuracy

\section{Discussion}

Yellow fever has re-emerged in Brazil, and is spreading into new regions. This range expansion is reflected in the most recent vaccine recommendation maps [14]. Accompanying this geographic expansion is an increase in the number of cases of YF, with large outbreaks in 2016/ 2017 and 2017/2018 (as of June 2018). Although a vaccine exists for the disease, understanding of the spatial and temporal risk of the spillover has been insufficient to predict where vaccines, a limited resource, should be distributed. We created retrospective time-varying risk maps of YF spillover based on environmental and demographic variables, identifying regions at risk of YF spillover throughout the year. In addition to the Amazon River basin, our maps highlight periods when the urban coastal region, a densely-populated area at the edge of YF expansion, is at an increased risk of disease spillover from NHP reservoirs. This approach can be expanded to incorporate near real-time data for risk mapping and decision support.

While most municipalities did not experience a long-term increase or decrease in intensity of spillover over the study period, those that did increase are in the southeastern and northern regions of Brazil. This agrees with other studies that have found YF virus to be spreading towards the southeast region in the past two decades, expanding from the enzoonotic zone to the transition zone $[2,39]$. Our model found municipalities to have the highest predicted intensity of YF spillover in 2008, which corresponds to a large outbreak in Brazil's southern states during that year [40]. Interestingly, we found the predicted intensity of a yellow fever spillover event to increase in July and August from 2001 to 2013, suggesting a lengthening of the season during which yellow fever is transmitted. However, this may also be an artefact of the time period used in our analysis, which ended with multiple consecutive La Niña years. La Niña years are characterized by a lengthening of the rainy season [41], during which vectors of YFV may be more abundant, leading to higher incidence of spillover into human populations. Regional climate cycles have been linked to transmission of vector-borne diseases elsewhere $[29,42,43]$, and it is likely that changes in precipitation and temperature resulting from La Niña and El Niño events drive YF spillover in Brazil as well.

By modeling regional spillover risk separately in high and low NHP richness regions, we identified different seasonal patterns between regions. The largest difference 


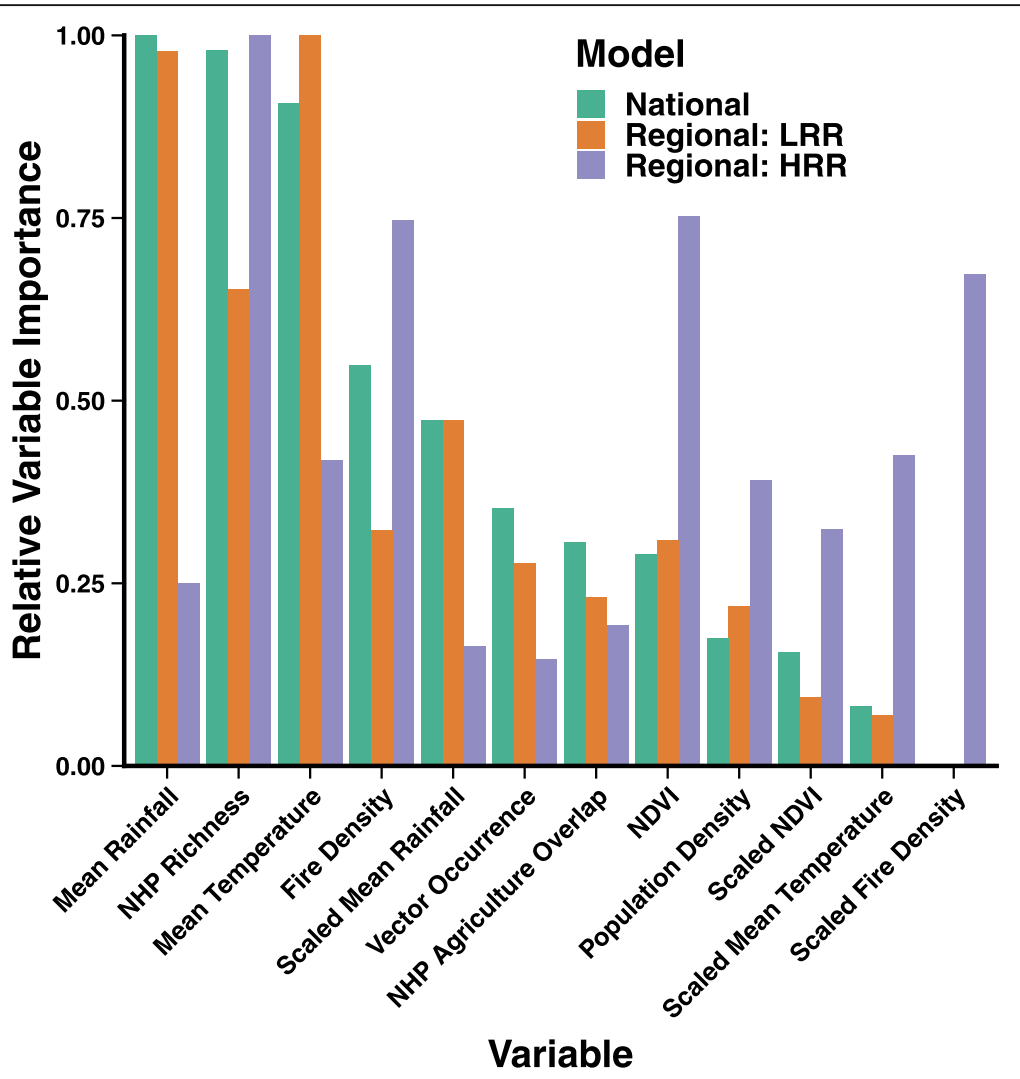

Fig. 6 Variable importance for the National and Regional model. The median variable importance was calculated for the National, low reservoir richness (LRR), and high reservoir richness (HRR) Regional models based on 100 permutations per variable within a model. Values are the decline in $A \cup C(\triangle A \cup C)$ due to permutation from the original model scaled to the largest $\triangle A U C$ within each model

in seasonal patterns between the National and Regional model is in the Amazon River basin, which shifts from a consistently high risk area in the National model (Fig. 4a) to alternating between high and low risk through the year in the Regional model (Fig. 4c). We found different patterns in the seasonal peaks of high spillover propensities when comparing between the LRR and HRR regions within the Regional model. This asynchrony of peak spillover propensity implies that at given times in the year, spillover risk can be higher in the urban, coastal regions than the Amazon River basin. This is contrary to contemporary maps of YF distribution in Brazil [14], but is consistent with the geographic distribution of the recent outbreaks of YF in 2016-2018.

There was a stark difference in the variables driving spillover in the National and LRR Regional models (which had a similar ranking of variable importance), and the HRR Regional model (Fig. 5). For the National and LRR Regional models, rainfall, temperature, and NHP species richness were the three most important variables. These variables were chosen a priori to represent vector and reservoir dynamics, and their ranking suggests that it is the combination of the presence of NHP reservoirs and high environmental suitability for mosquito vectors that leads to YF spillover. Other mosquito-borne diseases in South America are strongly correlated with climatic variables $[38,44,45]$. Similarly, we find evidence that YF spillover events are driven by temperature and rainfall in the LRR region. However, rainfall is less important in the HRR Regional model. Unlike the LRR region, the HRR region is composed entirely of Amazon rainforest, which receives over $4 \mathrm{~m}$ of rainfall annually and has low potential evaporation rates [46], allowing standing water, which serves as mosquito habitat, to remain throughout the year. Although the onset of the rainy season in the Amazon can lead to an increase in the abundance of YFV vectors [47], this is dependent on species, and vectors of YF persist year round [48]. In the HRR region, therefore, it is likely that vector populations are not strongly limited by rainfall, supporting our results. This context-dependence of the effect of individual environmental variables on mosquitoborne disease has been shown for malaria [49] and should be further explored for the case of YF.

Further, landscape characteristics such as NDVI and fire density are more informative than climatic variables in the HRR region of the Regional model. NDVI is a measure of vegetative cover that, in Brazil, is a lagged response to global climate that represents seasonal climate conditions 
corresponding to the wet and dry season $[50,51]$. In this way, it can be thought of as another climatic variable. In addition to climate, NDVI values are strongly influenced by land clearing techniques that rely on fire [51]. Given the relationship between NDVI and fire-fallow agriculture, and the importance of fire density in the HRR Regional model, human modification of the landscape via deforestation could be driving spillover events in this region. Malaria prevalence and the abundance of anopheline species often increases following human migration and conversion of land from forest for agricultural development in the Amazon [52,53] and spillover of another vector-borne disease of NHP origin, Plasmodium knowlesi, is driven by deforestation in Southeast Asia [54]. Similarly, human encroachment into and conversion of NHP habitat may be driving YF spillover from NHP to humans in the Amazon basin.

While the boundary separating high and low NHP reservoir richness regions was based on data, there may be scope for further development of this feature of the model. Specifically, the different seasonality of spillover risk between the regions highlights the need to study individual NHP species' ability to transmit YFV. This is particularly important given that potential non-human primate reservoirs are present in all but 21 municipalities in Brazil. The NHP species included in the model are from genera known to be susceptible to YF as detected by active infection or antibody titers $[3,16,55]$. However, little is known about individual species' competencies, which is especially important given the known role of highly competent species in sustaining transmission amongst reservoirs of other vector-borne diseases [56-58]. In addition to competency, data on the relative abundance of NHP populations and mosquitoes' biting preferences would help identify NHP species most likely to contribute to YF spillover. A better understanding of species' competencies and ability to transmit YFV to mosquitoes would narrow down the current list of NHP reservoirs to those which genuinely play a role in viral transmissions. This could identify other municipalities without a 'true' NHP reservoir present, and therefore not at risk of YF spillover. Future work should address the variation in reservoir competency between species, and the impact of NHP community composition on sylvatic transmission.

Our logistic bagging approach performs well given that the dataset was extremely sparse, with municipalities reporting spillover events approximately $0.01 \%$ of the time. The lower testing AUC for the HRR Regional model (AUC $=0.63$ ) is likely due to the sparse dataset. This region only had 20 spillover events during the 13 year period, which was further split into training and testing observations (Table 2). The model performance is based on accurately classifying five presences from the 9926 background points.
Logistic bagging may be useful in other situations where over fitting models to sparse data is a concern, such as St. Louis encephalitis, Lassa fever, or Rift Valley fever. Additionally, a binary response variable can be more robust than a continuous response variable. First, a binary response buffers against gradual improvements in surveillance practices, which is most likely the case for YF in Brazil, since single and multiple cases per an observational unit are equally weighted. This is supported by the lack of an overall increase in risk over the study period. Additional precaution, however, would be needed if a surveillance practices were to dramatically change. For example, this approach would not be suitable to handle case data spanning the implementation of surveillance infrastructure development completed in the early 2000s [59]. Secondly, for the sylvatic YF spillover system, the logistic response variable avoids classifying cases resulting from sylvatic and urban transmission. While the most recent YF outbreaks in Brazil may include cases resulting from both urban and sylvatic transmission, it is doubtful that urban transmission occurred during our study (2001-2013) [5].

This approach is limited by the availability of environmental and epidemiological data that are both continuous and have high temporal and spatial resolution. For neglected diseases, epidemiological data at the sub-administrative level is not always available nor current. Further, remotely sensed environmental data are often at spatial resolutions that are coarser than the disease data, or are averaged over time. For example, daily variation in temperature is shown to have larger effects on mosquito dynamics than mean daily temperature alone [27], but satellite imagery collected daily is unable to measure such daily variation. Processing of satellite imagery is rarely standardized and is not continuous over time due to changes in satellite technology [60], making comparisons across datasets difficult [61]. This limitation is reflected in the current study, whose study period coincides with that of the availability of land cover data, preventing us from predicting YF spillover into the present.

\section{Conclusions}

The spillover risk of YF in Brazil varies in space and time. When spillover risk is modeled using separate models for different ecological contexts (i.e. low $v s$ high NHP species richness), differing seasonal patterns emerge. Specifically, when combining predictions across low and high non-human primate richness regions, the asynchrony of spillover seasonality can lead to intermittent hot spots of YF spillover along urban coastal areas, which coincide with 2016/ 2017 and 2017/2018 YF outbreaks. These seasonal patterns may be due to differences in environmental drivers across a large geographic area and should be further explored through ecological and entomological studies. Understanding these patterns of YF spillover could better 
inform allocation of public health services and resources, such as vaccination campaigns, which is particularly important given the current shortage in the YF vaccine [62]. Predictive models of disease spillover should incorporate fine-scale temporal and spatial resolutions to better approximate the ecological context and processes driving spillover events.

\section{Additional files}

Additional file 1: Text 1. Additional Methods. Methods for data collection, preliminary data exploration, and additional analyses of results. Table S1. Results of univariate analyses. Mean AUC values ( \pm SD) of univariate bagged logistic regression models on training dataset using spatial minima, mean, or maxima of rainfall and temperature. Figure S1. Correlation matrix of full set of covariates. Numeric inset represents Pearson correlation coefficient. Highly correlated covariates were not included in the models. Figure S2. Histogram of number of cases reported in municipality-month. The number of cases reported per municipality-month ranged from 0-18. Municipality-months without cases were not plotted. Figure S3. Histogram of municipalities with reoccurring spillover events. Figure S4. Long-term trends in predicted YF spillover. Results of slope tests exploring the change in predicted spillover intensity in each municipality across all 156 months. Values represent average change over all 156 months. Non-significant (alpha $=0.05$ ) slopes are reported as zero. Figure S5. Long-term trends in predicted YF spillover by calendar month. Results of slope tests exploring the change in predicted spillover intensity in each municipality and month of the year from 2001 to 2013. Values represent average yearly change for each month. Non-significant (alpha $=0.05$ ) slopes are reported as zero. (PDF 3636 kb)

Additional file 2: Predicted spatial risk of yellow fever. Complete time series of predictions. Black dots indicate a municipality reporting any YF cases. (GIF $6840 \mathrm{~kb}$ )

\section{Abbreviations}

HRR: High reservoir richness; LRR: Low reservoir richness; NDVI: Normalized Difference Vegetation Index; NHP: Non-human primate; SD: Standard deviation; YF: Yellow fever; YFV: Yellow fever virus

\section{Acknowledgements}

The authors would like to thank J. P. Schmidt for helpful insight during the project's conception. S. Hall and A. Schatz were essential for the timely completion of this work by assisting in data collection and optimizing code for the analysis. E. Marty created the conceptual diagram used in Fig. 1. The manuscript was greatly improved by comments from anonymous reviewers.

\section{Availability of data and materials}

The datasets generated and/or analyses associated with this study are available in the Figshare repository, 10.6084/m9.figshare.5985103.

\section{Authors' contributions}

The project was conceived by MVE and RBK. JMD, MVE and RBK designed the analysis. MVE and RBK performed the analysis and drafted manuscript. CM and JMD critically revised the manuscript. All authors read and approved the final manuscript.

\section{Ethics approval and consent to participate}

Not applicable.

\section{Consent for publication}

Not applicable.

\section{Competing interests}

The authors declare that they have no competing interests.

\section{Publisher's Note}

Springer Nature remains neutral with regard to jurisdictional claims in published maps and institutional affiliations.

\section{Author details}

${ }^{1}$ Center for the Ecology of Infectious Diseases, University of Georgia, Athens, GA, USA. ${ }^{2}$ Odum School of Ecology, University of Georgia, Athens, GA, USA. ${ }^{3}$ Department of Infectious Diseases, University of Georgia, Athens, GA, USA. ${ }^{4}$ Center for Tropical and Global Emerging Diseases, University of Georgia, Athens, GA, USA. ${ }^{5}$ Center for Vaccines and Immunology, University of Georgia, Athens, GA, USA. ${ }^{6}$ River Basin Center, University of Georgia, Athens, GA, USA.

Received: 24 April 2018 Accepted: 15 August 2018

Published online: 29 August 2018

\section{References}

1. Câmara FP, Carvalho D, Max L, Gomes ALB. Demographic profile of sylvatic yellow fever in Brazil from 1973 to 2008. Trans R Soc Trop Med Hyg. 2013; 107:324-7.

2. Vasconcelos PF d C. Yellow fever in Brazil: thoughts and hypotheses on the emergence in previously free areas. Rev Saude Publica. 2010:44:1144-9.

3. Moreno ES, Spinola R, Tengan CH, Brasil RA, Siciliano MM, Coimbra TLM, et al. Yellow fever epizootics in non-human primates, São Paulo State, Brazil, 2008-2009. Rev Inst Med Trop Sao Paulo. 2013;55:45-50.

4. Bicca-Marques JC, de Freitas DS. The role of monkeys, mosquitoes, and humans in the occurrence of a yellow fever outbreak in a fragmented landscape in south Brazil: protecting Howler monkeys is a matter of public health. Trop Conserv Sci. 2010;3:78-89.

5. Cavalcante KRLJ, Tauil PL, Cavalcante KRL, Tauil PL. Risk of re-emergence of urban yellow fever in Brazil. Epidemiol Serv Saúde. 2017;26:617-20.

6. Hay SI, Battle KE, Pigott DM, Smith DL, Moyes CL, Bhatt S, et al. Global mapping of infectious disease. Philos Trans R Soc B. 2013;368:20120250.

7. Schmidt JP, Park AW, Kramer AM, Han BA, Alexander LW, Drake JM. Spatiotemporal fluctuations and triggers of Ebola virus spillover. Emerg Infect Dis. 2017;23:415-22.

8. $\quad$ Pigott DM, Golding N, Mylne A, Huang Z, Henry AJ, Weiss DJ, et al. Mapping the zoonotic niche of Ebola virus disease in Africa. Elife. 2014;3:e04395-29.

9. Hay SI, Guerra CA, Gething PW, Patil AP, Tatem AJ. A world malaria map: Plasmodium falciparum endemicity in 2007. PLoS Med. 2009;6:e1000048.

10. Ryan SJ, McNally A, Johnson LR, Mordecai EA, Ben-Horin T, Paaijmans K, et al. Mapping physiological suitability limits for malaria in Africa under climate change. Vector-Borne Zoon Dis. 2015;15:718-25.

11. Machado-Machado EA. Empirical mapping of suitability to dengue fever in Mexico using species distribution modeling. Appl Geogr. 2012;33:82-93.

12. Bhatt $S$, Gething PW, Brady OJ, Messina JP, Farlow AW, Moyes CL, et al. The global distribution and burden of dengue. Nature. 2013;496:504-7.

13. Briand S, Beresniak A, Nguyen T, Yonli T, Duru G, Kambire C, et al. Assessment of yellow fever epidemic risk: an original multi-criteria modeling approach. PLoS Negl Trop Dis. 2009:3:e483.

14. Jentes ES, Poumerol G, Gershman MD, Hill DR, Lemarchand J, Lewis RF, et al. The revised global yellow fever risk map and recommendations for vaccination, 2010: consensus of the Informal WHO Working Group on Geographic Risk for Yellow Fever. Lancet Infect Dis. 2011:11:622-32.

15. Hamrick PN, Aldighieri S, Machado G, Leonel DG, Vilca LM, Uriona S, et al. Geographic patterns and environmental factors associated with human yellow fever presence in the Americas. PLoS Negl Trop Dis. 2017;11: e0005897.

16. Moreno ES, Agostini I, Holzmann I, Bitetti D, S M, Oklander LI, et al. Yellow fever impact on brown howler monkeys (Alouatta guariba clamitans) in Argentina: a metamodelling approach based on population viability analysis and epidemiological dynamics. Mem Inst Oswaldo Cruz. 2015;110:865-76.

17. Câmara FP, Gomes ALBB, de Carvalho LMF, Castello LGV. Dynamic behavior of sylvatic yellow fever in Brazil (1954-2008). Rev Soc Bras Med Trop. 2011; 44:297-9.

18. Kovats RS, Bouma MJ, Haines A. El Nino and Health. Geneva: World Health Organization; 1999.

19. Cavalcante KRLJ, Tauil PL, Cavalcante KRL, Tauil PL. Epidemiological characteristics of yellow fever in Brazil, 2000-2012. Epidemiol Serv Saúde. 2016;25:11-20. 
20. Bowden SE, Magori K, Drake JM. Regional differences in the association between land cover and West Nile virus disease incidence in humans in the United States. Am J Trop Med Hyg. 2011;84:234-8.

21. Rogers DJ, Wilson AJ, Hay SI, Graham AJ. The global distribution of yellow fever and dengue. Adv Parasitol. 2006;62:181-220.

22. Shearer FM, Longbottom J, Browne AJ, Pigott DM, Brady OJ, Kraemer MUG, et al. Existing and potential infection risk zones of yellow fever worldwide: a modelling analysis. Lancet Glob Health. 2018;6:e270-8.

23. Dark SJ, Bram D. The modifiable areal unit problem (MAUP) in physical geography. Prog Phys Geogr. 2007;31:471-9.

24. Hay GJ, Marceau DJ, Dubé P, Bouchard A. A multiscale framework for landscape analysis: object-specific analysis and upscaling. Landsc Ecol. 2001; 16:471-90.

25. Brady OJ, Johansson MA, Guerra CA, Bhatt S, Golding N, Pigott DM, et al Modelling adult Aedes aegypti and Aedes albopictus survival at different temperatures in laboratory and field settings. Parasit Vectors. 2013;6:351.

26. Weaver SC, Reisen WK. Present and future arboviral threats. Antivir Res. 2010;85:328-45.

27. Paaijmans KP, Heinig RL, Seliga RA, Blanford Jl, Blanford S, Murdock CC, et al. Temperature variation makes ectotherms more sensitive to climate change. Glob Chang Biol. 2013;19:2373-80.

28. Lambrechts L, Paaijmans KP, Fansiri T, Carrington LB, Kramer LD, Thomas $M B$, et al. Impact of daily temperature fluctuations on dengue virus transmission by Aedes aegypti. Proc Natl Acad Sci USA. 2011;108:7460-5.

29. Chuang T-W, Chaves LF, Chen P-J. Effects of local and regional climatic fluctuations on dengue outbreaks in southern Taiwan. PLoS One. 2017;12: e0178698.

30. Millones M, Rogan J, li BLT, Parmentier B, Harris RC, Griffith DA. Fire data as proxy for anthropogenic landscape change in the Yucatán. Land. 2017;6:61.

31. Jones KE, Patel NG, Levy MA, Storeygard A, Balk D, Gittleman JL, et al. Global trends in emerging infectious diseases. Nature. 2008:451:990-3.

32. Power AG, Mitchell CE. Pathogen spillover in disease epidemics. Am Nat. 2004;164:S79-89.

33. Keesing F, Holt RD, Ostfeld RS. Effects of species diversity on disease risk Ecol Lett. 2006;9:485-98.

34. Breiman L. Bagging predictors. Mach Learn. 1996;24:123-40.

35. Valentini G, Dietterich TG. Low bias bagged support vector machines. In: Proceedings of the 20th International Conference on Machine Learning (ICML-03); 2003. p. 752-9.

36. Grafström A, Lisic J. BalancedSampling: Balanced and Spatially Balanced Sampling. 2016. https://CRAN.R-project.org/package=BalancedSampling

37. Grafström A, Tillé Y. Doubly balanced spatial sampling with spreading and restitution of auxiliary totals. Environmetrics. 2013;24:120-31.

38. Stewart-lbarra AM, Muñoz ÁG, Ryan SJ, Ayala EB, Borbor-Cordova MJ, Finkelstein $J$, et al. Spatiotemporal clustering, climate periodicity, and social-ecological risk factors for dengue during an outbreak in Machala, Ecuador, in 2010. BMC Infect Dis. 2014;14:610

39. Vasconcelos PFC, Bryant JE, Travassos da Rosa APA, Tesh RB, Rodrigues SG, Barrett ADT. Genetic divergence and dispersal of yellow fever virus, Brazil. Emerg Infect Dis. 2004;10:1578-84.

40. Romano AP, Costa ZG, Ramos DG, Andrade MA, Jayme Vde S, Almeida MA, et al. Yellow fever outbreaks in unvaccinated populations, Brazil, 2008-2009. PLoS Negl Trop Dis. 2014;8:e2740

41. Marengo JA, Liebmann B, Kousky VE, Filizola NP, Wainer IC. Onset and end of the rainy season in the Brazilian Amazon Basin. J Clim. 2001;14:833-52.

42. Huang X, Clements ACA, Williams G, Devine G, Tong S, Hu W. El NiñoSouthern Oscillation, local weather and occurrences of dengue virus serotypes. Sci Rep. 2015;5:16806.

43. Stewart-lbarra AM, Lowe R. Climate and non-climate drivers of dengue epidemics in southern coastal Ecuador. Am J Trop Med Hyg. 2013;88:971-81.

44. Huber JH, Childs ML, Caldwell JM, Mordecai EA. Seasonal temperature variation influences climate suitability for dengue, chikungunya, and Zika transmission. PLoS Negl Trop Dis. 2018;12:e0006451.

45. Lowe R, Stewart-Ibarra AM, Petrova D, García-Díez M, Borbor-Cordova MJ, Mejía R, et al. Climate services for health: predicting the evolution of the 2016 dengue season in Machala, Ecuador. Lancet Planetary Health. 2017;1: e142-51.

46. Shuttleworth WJ. Evaporation from Amazonian rainforest. Proc R Soc Lond B. 1988;233:321-46.

47. Degallier N, Monteiro HADO, Castro FC, Silva OVD, Filho GCS, Elguero E. An indirect estimation of the developmental time of Haemagogus janthinomys
(Diptera: Culicidae), the main vector of yellow fever in South America. Studies Neotrop Fauna Env. 2006:41:117-22.

48. Pinto CS, Confalonieri UE, Mascarenhas BM. Ecology of Haemagogus sp. and Sabethes sp. (Diptera: Culicidae) in relation to the microclimates of the Caxiuanã National Forest, Pará, Brazil. Mem Inst Oswaldo Cruz. 2009;104:592-8.

49. Olson SH, Gangnon R, Elguero E, Durieux L, Guégan J-F, Foley JA, et al. Links between climate, malaria, and wetlands in the Amazon Basin. Emerg Infect Dis. 2009;15:659-62.

50. Zhao W, Zhao X, Zhou T, Wu D, Tang B, Wei H. Climatic factors driving vegetation declines in the 2005 and 2010 Amazon droughts. PLoS One. 2017:12:e0175379.

51. Gurgel HC, Ferreira NJ. Annual and interannual variability of NDVI in Brazil and its connections with climate. Int J Remote Sens. 2003;24:3595-609.

52. Yasuoka J, Levin R. Impact of deforestation and agricultural development on anopheline ecology and malaria epidemiology. Am J Trop Med Hyg. 2007; 76:450-60.

53. Conn JE, Wilkerson RC, Segura MNO, de Souza RTL, Schlichting CD, Wirtz RA, et al. Emergence of a new Neotropical malaria vector facilitated by human migration and changes in land use. Am J Trop Med Hyg. 2002;66:18-22.

54. Fornace KM, Abidin TR, Alexander N, Brock P, Grigg M, Murphy A, et al. Association between landscape factors and spatial patterns of Plasmodium knowlesi infections in Sabah, Malaysia. Emerg Infect Dis. 2016:22:201-8.

55. Moreno ES, Rocco IM, Bergo ES, Brasil RA, Siciliano MM, Suzuki A, et al. Reemergence of yellow fever: detection of transmission in the State of Sao Paulo, Brazil 2008. Rev Soc Bras Med Trop. 2011;44:290-6.

56. LoGiudice K, Ostfeld RS, Schmidt KA, Keesing F. The ecology of infectious disease: effects of host diversity and community composition on Lyme disease risk. Proc Natl Acad Sci USA. 2003;100:567-71.

57. Keesing F, Allan BF, Young TP. Effects of wildlife and cattle on tick abundance in central Kenya. Ecol Appl. 2013;23:1410-8.

58. Reisen WK, Chiles RE, Martinez VM, Fang Y, Green EN. Experimental infection of California birds with western equine encephalomyelitis and St. Louis encephalitis viruses. J Med Entomol. 2003:40:968-82.

59. World Bank. Brazil - Disease Surveillance and Control Project (VIGISUS). World Development Sources, WDS 1998-3. Washington, DC: World Bank. http://documents.worldbank.org/curated/en/165871468743369557/BrazilDisease-Surveillance-and-Control-Project-VIGISUS

60. Janetos AC, Justice CO. Land cover and global productivity: a measurement strategy for the NASA programme. Int J Remote Sens. 2000;21:1491-512.

61. Bai $Y$, Feng $M$, Jiang H, Wang J, Zhu Y, Liu Y. Assessing consistency of five global land cover data sets in China. Remote Sens-Basel. 2014;6:8739-59.

62. Gershman MD. Addressing a yellow fever vaccine shortage - United States, 2016-2017. MMWR Morb Mortal Wkly Rep. 2017;66:457-9.

Ready to submit your research? Choose BMC and benefit from:

- fast, convenient online submission

- thorough peer review by experienced researchers in your field

- rapid publication on acceptance

- support for research data, including large and complex data types

- gold Open Access which fosters wider collaboration and increased citations

- maximum visibility for your research: over $100 \mathrm{M}$ website views per year

At $\mathrm{BMC}$, research is always in progress.

Learn more biomedcentral.com/submissions 